\title{
Centre Manifold of Fractional-order Lorenz system
}

\author{
Yanling Guo ${ }^{1}$ and Yanxia Sun ${ }^{2+}$ \\ ${ }^{1}$ F’SATIE, Department of Electrical Engineering, Tshwane University of Technology, Pretoria 0001, South \\ Africa \\ ${ }^{2}$ Department of Electrical and Electronic Engineering Science, University of Johannesburg, Johannesburg \\ 2006, South Africa
}

\begin{abstract}
Dimension reduction is a significant method of simplifying the study to reduce the dimensions of a dynamic system for technical applications in dimensional systems of science or engineering. Centre Manifold method is a mathematical prescription of reduction method in ordinary differential systems. In this paper, a local fractional centre manifold is introduced and applied to the fractional order Lorenz system.
\end{abstract}

Keywords: fractional dynamical system, fractional order Lorenz system, fractional centre manifold, Caputo derivative.

\section{Introduction}

The fractional calculus was first mentioned in in 1695 by Leibniz and L'Hospital. At the end of the 19-th century, Liouville and Riemann introduced the first definition of the fractional derivative [14]. In recent years, fractional calculus has attracted a number of authors for describing many mathematical problems in physics and engineering modelled by fractional differential equations [16]. According to variational principle, it is possible to construct a complete mechanical description of non-conservative system [12]. In 2002, the electrochemical processes and flexible robot arm are modelled by fractional order models [18]. A great number of fractional order model are found in Brownian motion, rheology, viscoelasticy, thermodynamics [18]

Centre Manifold method is a rigorous formulation of this observation and it usually allows one to reduce a large problem to a much smaller one. The first rigorous outputs on invariant manifolds were presented by Hadamard (1901) [5], Lyapunov(1907) [7] and Perron (1929) [10]. They proved the existence of stable and unstable manifolds for systems of differential equations and maps. As a significant reduction method, the centre manifold theory was first proposed in 1960s [11]. As an essential tool, it is applied for investigation of dynamics, such as bifurcation, stability, and perturbation [1, 4, 17].

In this paper, first, some basic definition about fractional calculus are introduced, second, the centre Manifold of Fractional Dynamical System is deduced, and the unstable invariant manifold, stable invariant manifold and centre manifold are discussed. In the end, with some condition, the centre Manifold of fractional Lorenz system is analysed.

\section{Definition of Fractional System}

Definition 1 (Induced Matrix Norms [9]. A vector norm, defined on $C^{p}$ for $p=m, n$, induces a matrix norm on $C^{m \times n}$ by

$$
\|A\|=\max _{\|x\|=1}\|A x\| \text { for } A \in C^{m \times n}, x \in C^{n \times 1} .
$$

\footnotetext{
+ Corresponding author. Tel.: + 27767916927;

E-mail address: sunyanxia@ gmail.com.
} 
Definition 2 (Matrix 2-norm) the matrix 2-norm [9] is

$$
\|A\|=\|A\|_{2}=\max _{\|x\|_{2}=1}\|A x\|_{2}=\sqrt{\zeta_{\max }}
$$

Here $x \in R^{n},\|x\|=\left(\sum_{i=1}^{n} x_{i}^{2}\right)^{\frac{1}{2}}, A \in R^{n \times n}$, and $\zeta_{\max }$ is the largest number $\zeta$ such that $A^{T} A-\zeta I$ is singular, $I \in R^{n \times n}$ is diagonal matrix from the Eigen decomposition of $A$.

Definition 3(The Euler Gamma function) The Euler Gamma function $\Gamma$ is defined for $x>0$ as

$$
\Gamma(x)=\int_{0}^{\infty} e^{-t} t^{x-1} d t
$$

Theorem 1 for all $a \geq 1$, the function [13]

$$
f(x)=\frac{\Gamma(1+x)^{a}}{\Gamma(1+a x)}
$$

is a decreasing function of $x \geq 0$.

Corollary 1 for all $a \geq 1$ and $x \in[0,1]$,

$$
\frac{1}{\Gamma(1+a)} \leq \frac{\Gamma(1+x)^{a}}{\Gamma(1+a x)} \leq 1
$$

Definition 4 (Real Mittag-Leffter function [15], The function $E_{\alpha}: R \rightarrow R$ defined by

$$
E_{\alpha}(x)=\sum_{i=0}^{\infty} \frac{x^{i}}{\Gamma(\alpha i+1)}, x \in R, \alpha>0
$$

$E_{\alpha}$ can be wrote as follows

$$
E_{\alpha}(x)=\sum_{i=0}^{m} \frac{x^{i}}{\Gamma(\alpha i+1)}+\sum_{i=m+1}^{\infty} \frac{x^{i}}{\Gamma(\alpha i+1)},
$$

From corollary $1 \Gamma(1+\alpha i) \geq 1$ for all $\alpha i \geq 1$. Let $i_{0}=1+[1 / \alpha]$, here [·] is a floor function, which is the largest integer not bigger than $x$.

If $m+1 \geq i_{0}$

$$
\left|\sum_{i=m+1}^{\infty} \frac{x^{i}}{\Gamma(\alpha i+1)}\right| \leq \sum_{i=m+1}^{\infty}|x|^{i}=\frac{|x|^{m+1}}{1-|x|} .
$$

If $i_{0} \geq \ln (\rho(1-|x|)) / \ln (|x|)$ and $|x|<1$, then $\left|\sum_{i=i_{0}+1}^{\infty} x^{i} / \Gamma(\alpha i+1)\right| \leq \rho$.

Lemma 1 When $|x| \leq q, 0<q<1,0<\alpha<1$, the Mittag-Leffler function is showed with $\rho>0$

$$
E_{\alpha}(x)=\sum_{i=0}^{i_{0}} \frac{x^{i}}{\Gamma(\alpha i+1)}+\sum_{i=i_{0}+1}^{\infty} \frac{x^{i}}{\Gamma(\alpha i+1)}, \quad\left|\sum_{i=i_{0}+1}^{\infty} \frac{x^{i}}{\Gamma(\alpha i+1)}\right| \leq \rho
$$

Definition 5 (Caputo Fractional derivative) The Caputo Fractional derivative [15] with order $\alpha\left(n-1<\alpha<n, n \in Z^{+}\right)$is defined as

$$
{ }_{c} D_{0, t}^{\alpha} f(t)=\frac{1}{\Gamma(\alpha)} \int_{0}^{t}(t-\tau)^{n-\alpha-1} f^{(n)}(\tau) d \tau
$$

\section{Centre manifold of Fractional Dynamical System}

Consider an autonomous fractional ordinary differential system,

$$
{ }_{c} D_{0, t}^{\alpha} x(t)=w(x), \quad \alpha \in(0,1), \quad x \in R^{n+m}
$$

where $n, m \in N^{+}, \quad N^{+}$is positive integer. There are three hypnoses for the centre manifold: $x=0$ i.e. $w(0)=0$ is an equilibrium point of Eq. 11; The eigenvalues of $w_{x}^{\prime}(0)$ are nonzero eigenvalue;

The arguments of the eigenvalues $\left|\arg \left(\lambda\left(w_{x}^{\prime}(0)\right)\right)\right| \geq \alpha \pi / 2$

Based on these assumption, the system (Eq. 11) can be transformed into 


$$
\begin{aligned}
& { }_{c} D_{0, t}^{\alpha} y(t)=A y+f(y, z), \\
& { }_{c} D_{0, t}^{\alpha} z(t)=B z+g(y, z) .
\end{aligned}
$$

where $y \in R^{n}, z \in R^{m}, \alpha \in(0,1)$ and $A \in R^{n \times n}, B \in R^{m \times m}$ are the linearized matrices.

Remark 1 if there exist eigenvalues of $f_{x}^{\prime}(0)$, whose arguments $\left|\arg \left(\lambda\left(f_{x}^{\prime}(0)\right)\right)\right|<\alpha \pi / 2$, the spectrum $E=f_{x}^{\prime}(0)$ is divided into $\sigma_{u}, \sigma_{s}, \sigma_{c}$ three parts

$$
|\arg \lambda(E)| \begin{cases}<\alpha \pi / 2, & \text { if } \lambda \in \sigma_{u} \\ >\alpha \pi / 2, & \text { if } \lambda \in \sigma_{s} \\ =\alpha \pi / 2, & \text { if } \lambda \in \sigma_{c}\end{cases}
$$

Let $E^{u}, E^{s}, E^{c}$ be the unstable, stable, center subspace of $\sigma_{u}, \sigma_{s}, \sigma_{c}$, then $W^{u}, W^{s}$ and $W^{c}$ are unstable invariant manifold, stable invariant manifold and center manifold which tangent to $E^{u}, E^{s}, E^{c}$ at equilibrium point

When $\alpha=1,|\arg (\lambda)|=\pi / 2$, then the stable region is the eigenvalue with negative real part. It is the same with the centre manifold of ordinary differential systems.

Definition 6 (a local invariant manifold of fractional ordinary differential) consider a fractional ordinary differential system

$$
{ }_{c} D_{0, t}^{\alpha} x(t)=w(x) .
$$

If $x_{0} \in S \subseteq R^{n}, x(t) \in S$, for $t<\delta$. Here $x_{0}$ is the initial of Eq. 4.16 i.e. $x(0)=x_{0}, x(t)$ is the solution of Eq. $14, \delta$ is a suitably small positive number.

Definition 7 (a local centre manifold of fractional ordinary differential system [8]) if a local invariant manifold $y=h(x)$ of Eq. 12 satisfy $h(0)=0, h^{\prime}(0)=0$, the solution $(x(t), y(t))$ of Eq. 12, with initial condition $\left(x_{0}, y_{0}\right)=\left(x_{0}, h\left(x_{0}\right)\right)$, remain on the manifold $y=h(x)$ for sufficiently small $t$, it can be called a local centre manifold of fractional ordinary differential system.

Theorem 4.2 There is a fractional manifold centre $y=h(x)$ in system (12), for $|x|<\theta, \theta>0, h \subset C^{2}$.

\section{Centre Manifold of Fractional-Order Lorenz System}

Let $f^{\alpha}=d^{\alpha} / d t^{\alpha}$, Consider the fractional-order Lorenz system(3)

$$
\begin{aligned}
& f^{\alpha}(x)=\sigma(y-x), \\
& f^{\alpha}(y)=\rho x-y-x z, \\
& f^{\alpha}(z)=-b z+x y .
\end{aligned}
$$

The linearized system of Eq. 15 at $O(0,0,0)$

$$
\begin{aligned}
& f^{\alpha}(x)=a(y-x) \\
& f^{\alpha}(y)=c x+d y, \\
& f^{\alpha}(z)=-b z .
\end{aligned}
$$

The Jacobin of Eq. 15 at $O(0,0,0)$ is

$$
J=\left[\begin{array}{ccc}
-\sigma & \sigma & 0 \\
\rho & -1 & 0 \\
0 & 0 & -b
\end{array}\right]
$$

Then the characteristic equation

$f(\lambda)=(\lambda+b)\left[\lambda^{2}+(1+\sigma) \lambda+\sigma-\sigma \rho\right]$
Therefore, eigenvalues of Eq. 15 are $\lambda_{1}=-b, \lambda_{2,3}=-(1+\sigma) / 2 \pm \sqrt{(1+\sigma)^{2}+4 \sigma(\rho-1)} / 2$

If $\sigma<-1$, and $\rho<1-(1+\sigma)^{2} / 4 \sigma, \sqrt{(1+\sigma)^{2}+4 \sigma(\rho-1)} / 2$ is an imaginary number, $\lambda_{2}$ and $\lambda_{3}$ are a pair of roots with positive real parts.

Since $b \in R^{+}$, then $\left|\arg \left(\lambda_{1}\right)\right|=\pi>\alpha \pi / 2$, for $0<\alpha<1$.

According to theorem 2, exists a fractional centre manifold $h(x)$ for Eq. 15, if $\left|\arg \left(\lambda_{2}\right)\right|=\left|\arg \left(\lambda_{3}\right)\right|=\alpha \pi / 2$, i.e. $\tan (\alpha \pi / 2)=\sqrt{-(1+\sigma)^{2}-4 \sigma(\rho-1)} /(-1-\sigma)$, When $\alpha=1 / 2, \rho=(1+\sigma)^{2} /(2 \sigma)$. 
But it is difficult to get the analytical expression of the centre manifold, according to theorem 4.3 a function is defined to approximate $h(X)$

$$
M \phi(X)={ }_{c} D_{0, t}^{\alpha} \phi(X)+b \phi(X)-x y
$$

here $X=(x(t), y(t))^{T}$.

Suppose

$$
\phi(X)=a_{1} x^{2}(t)+a_{2} y^{2}(t)=O\left(X^{2}\right)
$$

where $a_{1}$ and $a_{2}$ are undetermined coefficients.

Let $x(t)$ and $y(t)$ be analytic function on $[0, \delta]$

$$
\begin{aligned}
& x(t)=x(0)+x^{\prime}(0) t+x^{\prime \prime}(0) t^{2} / 2+O\left(|t|^{3}\right) \\
& y(t)=y(0)+y^{\prime}(0) t+y^{\prime \prime}(0) t^{2} / 2+O\left(|t|^{3}\right)
\end{aligned}
$$

According to the property of Caputo derivate [15] with the initial condition $x(0)=y(0)=0$ and

$$
\begin{aligned}
{ }_{c} D_{0, t}^{1 / 2} \phi(X) & =D_{0, t}^{1 / 2}\left[a_{1} x^{2}(t)+a_{2} y^{2}(t)\right]=D_{0, t}^{1 / 2}\left[a_{1}\left(x^{\prime}(0) t+x^{\prime \prime}(0) t^{2} / 2+O\left(|t|^{3}\right)\right)+a_{2}\left(y^{\prime}(0) t+y^{\prime \prime}(0) t^{2} / 2+O\left(|t|^{3}\right)\right)\right] \\
& =\left(2 a_{1} x^{\prime}(0) / \Gamma(5 / 2)+2 a_{2} y^{\prime}(0) / \Gamma(5 / 2)\right) t^{3 / 2}+O\left(|t|^{5 / 2}\right)
\end{aligned}
$$

In order to get ${ }_{c} D_{0, t}^{1 / 2} \phi(x)=O\left(|t|^{5 / 2}\right)=O\left(|x|^{5 / 2}\right)$, the suitable coefficients $a_{1}=2$ and $a_{2}=3$ are selected to make $2 a_{1} x^{\prime}(0) / \Gamma(5 / 2)+2 a_{2} y^{\prime}(0) / \Gamma(5 / 2)=0$ and $a_{1}^{2}+a_{2}^{2} \neq 0$. Then the function $\phi(X)=O\left(|X|^{2}\right)=2 x^{2}+3 y^{2}$ is got.

Then

$$
M \phi(X)={ }_{c} D_{0, t}^{\alpha} \phi(X)+b \phi(X)-x y=O\left(|X|^{5 / 2}\right)+b O\left(|X|^{2}\right)-x y
$$

From $\phi(X)=O\left(|X|^{2}\right)=2 x^{2}+3 y^{2} \geq 6 \times 2 x y \quad, \quad x y \leq \phi(X) / 12=O\left(|X|^{2}\right) \quad, \quad$ therefore $\quad x y=O\left(|X|^{2}\right) \quad$, $M \phi(X)=O\left(|X|^{5 / 2}\right)$

According to theorem 3, the approximation of fractional centre manifold $h(X)=O\left(|X|^{5 / 2}\right)=2 b x^{2}+3 b y^{2}-x y$ at the neighbour of equilibrium point as shown in fig. $1 . b$ is the parameter of the system 15 , for easy plotting, we set $b=30$, then $h(X)=O\left(|X|^{5 / 2}\right)=60 x^{2}+90 y^{2}-x y$.

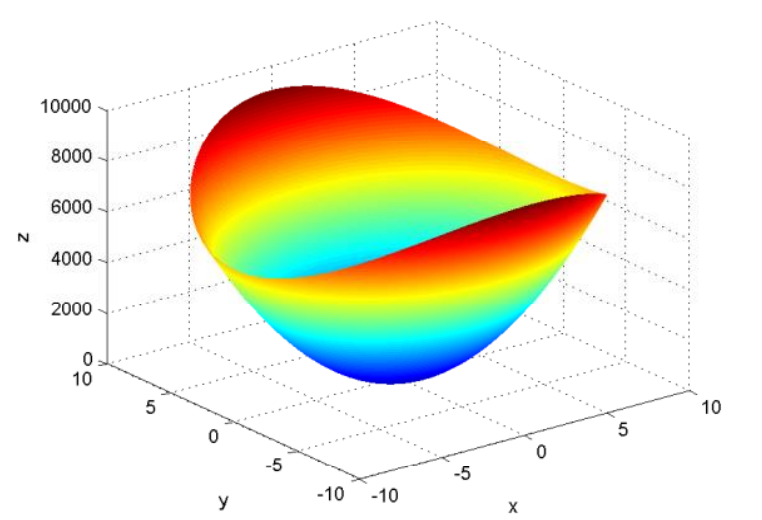

Fig. 1: The approximation of fractional centre manifold for system 15

\section{Conclusion}

In this paper, fractional centre manifold for fractional differential systems is introduced with the one of fractional order definition-Caputo derivative. Then, it is applied to analysis the property of the fractional differential Lorenz system, when the order $\alpha=1 / 2$.

\section{Acknowledgements}

This work was supported by South African National Research Foundation Incentive Grants (No. 81705 and 95687). 


\section{References}

[1] J. Carr. Applications of centre manifold theory, Springer Science \& Business Media, 2012.

[2] R. Gorenflo, A. A. Kilbas, F. Mainardi, S. V. Rogosin, Mittag-Leffler functions, related topics and applications, Springer, 2014.

[3] I. Grigorenko, E. Grigorenko. Chaotic dynamics of the fractional Lorenz system. Physical review letters, 2003, 91: 034101.

[4] J. Guckenheimer, P. J. HolmesNonlinear oscillations, dynamical systems, and bifurcations of vector fields, Springer Science \& Business Media, 2013.

[5] J. Hadamard.. Sur l'itération et les solutions asymptotiques des équations différentielles. Bull. Soc. Math. France, 1901, 29:224-228.

[6] A. Kelley. The stable, center-stable, center, center-unstable, unstable manifolds. Journal of Differential Equations, 1967, 3: 546-570.

[7] A. Liapounoff. Problème général de la stabilité du mouvement. Annales de la Faculté des Sciences de Toulouse, 1907. Université Paul Sabatier, 203-474.

[8] L. Ma, C. Li. Center manifold of fractional dynamical system. Journal of Computational and Nonlinear Dynamics, 2016, 11:021010.

[9] C. D. Meyer. Matrix analysis and applied linear algebra, Siam, 2000.

[10] O. erron, Über stabilität und asymptotisches verhalten der integrale von differentialgleichungssystemen. Mathematische Zeitschrift, 1929, 29:129-160.

[11] V. A. Pliss. A reduction principle in the theory of stability of motion. Izvestiya Rossiiskoi Akademii Nauk. Seriya Matematicheskaya, 1964, 28:1297-1324.

[12] F. Riewe. Mechanics with fractional derivatives. Physical Review E, 1997, 55:3581.

[13] J. Sandor. A note on certain inequalities for the gamma function. J. Ineq. Pure Appl. Math, 2005, 6.

[14] D. Sierociuk, A. Dzielinski. Fractional Kalman filter algorithm for the states, parameters and order of fractional system estimation. International Journal of Applied Mathematics and Computer Science, 2006, 16:129.

[15] H. M. Srivastava, J. J. Trujillo. Theory and applications of fractional differential equations. Elsevier, Amsterdam, 2006.

[16] P. J. Torvik, R. L. Bagley. On the appearance of the fractional derivative in the behavior of real materials. Journal of Applied Mechanics, 1984, 51:294-298.

[17] A. Vanderbauwhede. Centre manifolds, normal forms and elementary bifurcations. Dynamics reported. Springer, 1989.

[18] B. M. Vinagre, C. Monjie, A. Calderon. Fractional order systems and fractional order control actions. IEEE Conference on Decision and Control, 2002: 2550-2554.

[19] B. J. West, M. Bologna, P. Grigolini. Physics of fractal operators, Springer, 2003. 